\title{
Heart Failure Society of South Africa (HeFSSA) perspective on the European Society of Cardiology (ESC) 2012 chronic heart failure guideline
}

\author{
M T Mpe, MB ChB, FCP, MMed; E Q Klug, MB BCh, FCP, MMed; K S Sliwa, MD, PhD, FESC, FACC; J Hitzeroth, MB BCh, FCP; \\ D A Smith, MB BCh, FCP; on behalf of the Heart Failure Society of South Africa \\ You are encouraged to read the complete guideline: \\ McMurray JJV, Adamopoulos S, Anker SD, et al.; on behalf of the European Society of Cardiology. ESC guidelines for the diagnosis \\ and treatment of acute and chronic heart failure 2012. Eur Heart J 2012;33(14):1787-1847. [http://dx.doi.org/10.1093/eurheartj/ehs104] \\ Disclaimer: This guideline does not override the health professional's responsibility to make appropriate decisions according to the \\ circumstances of the patient. It is also the health professional's responsibility to verify the locally approved indications for the drugs and \\ devices mentioned in this guideline.
}

\begin{abstract}
Background. The South African Heart Association (SA Heart) is an affiliate of the European Society of Cardiology (ESC). SA Heart endorses ESC treatment guidelines with modification to suit local circumstances. The Heart Failure Society of South Africa (HeFSSA) is a special interest group of SA Heart. This guideline has been compiled on behalf of the HeFSSA and is based on the ESC guidelines for the diagnosis and treatment of acute and chronic heart failure 2012. The focus is on heart failure with reduced ejection fraction (HF-REF) (i.e. ejection fraction $<50 \%$ ). We have recommended interventions in symptomatic patients with HF-REF in general to clarify the 'grey area' between the ESC guidelines definition of $\operatorname{REF}(<50 \%)$ and the predefined ejection fraction used in randomised heart failure trials $(<35 \%)$.

Objective. To highlight new changes in the diagnosis and treatment of chronic heart failure with particular emphasis on areas that are relevant to SA. Conclusions. Randomised clinical trials are a crucial, but not the only, guide in treating HF-REF patients. There always remain questions that are unanswered and groups of patients not studied, so prudent clinical decisions are required.
\end{abstract}

S Afr Med J 2013;103(9 Suppl 2):661-667. DOI:10.7196/SAMJ.7319

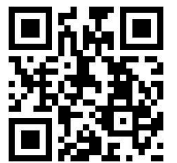

\section{Introduction}

The principal changes from the European Society of Cardiology (ESC) chronic heart failure (HF) 2008 guidelines relate to: $(i)$ new definition of HF with reduced ejection fraction (HF-REF); (ii) expanded indication for mineralocorticoid (aldosterone) receptor antagonists (MRAs); (iii) indication for the sinus node inhibitor ivabradine; (iv) expanded indication for cardiac resynchronisation therapy (CRT); $(v)$ new information on the role of coronary revascularisation; and $(v i)$ the role of ventricular assist devices.

\section{Objective}

The aim of this document is to highlight new changes with particular emphasis on areas that are relevant to South Africa.

\section{Definition and diagnosis of HF-REF} This guideline focuses on HF-REF; this term will replace the old reference 'left ventricular systolic dysfunction'. The new cut-off ejection fraction (EF) for HF-REF is a left ventricular (LV) EF $\leq 50 \%$.

Randomised HF-REF trials have used an entry LVEF $<35 \%$. Most of the medications indicated in HF-REF, have been studied in trials where the EF was $<35 \%$. The definition of HF-REF, however, is an $\mathrm{EF}<50 \%$. To avoid confusion between the inclusivity of the definition and the exclusivity of the randomised trials, we have taken a different approach to that of the ESC guideline. ${ }^{[1]}$ We have recommended the use of these agents in symptomatic patients with HF-REF in general. The ESC guideline states that those patients with an EF of $35-50 \%$ are in a 'grey area', but taking into account the way clinical medicine is practised, the inherent variability in $\mathrm{EF}$ assessments, and the need to treat symptomatic patients with an EF in the abnormal range, this document recognises the clinical need for more definitive recommendations. Randomised clinical trials are a crucial, but not the only, guide in treating HF-REF patients. There always remain questions that are unanswered and groups of patients not studied, so prudent clinical decisions are required.

\subsection{Clinical definition}

HF-REF is hereby defined as a syndrome in which patients have typical symptoms and signs resulting from an abnormality of LV function. It needs to be emphasised that HF is a syndrome and not a final diagnosis. Once the diagnosis of HF-REF has been made, it is important to establish the cause, in particular, a reversible cause, as well as a possible precipitating factor.

\subsection{Diagnosis}

3.2.1 Medical history

The patient's medical history is important. The index of suspicion for HF-REF is increased in patients with suggestive symptoms and a prior history of hypertension, coronary artery disease, chemotherapy and in peri-partum women. Importantly, HF-REF can present in patients with no prior relevant history.

\subsubsection{Symptoms}

Typical symptoms are breathlessness at rest or on exercise, fatigue, ankle and body swelling, orthopnoea, paroxysmal nocturnal dyspnoea and palpitations. 
Table 1. New York Heart Association classification of HF

\begin{tabular}{ll}
\hline Class & Description \\
\hline I & $\begin{array}{l}\text { Patient with cardiac disease, but no limitation on } \\
\text { ordinary physical activity }\end{array}$ \\
II & $\begin{array}{l}\text { Comfortable at rest, ordinary activity results in } \\
\text { symptoms (slight limitation) }\end{array}$ \\
III & $\begin{array}{l}\text { Comfortable at rest, less than ordinary activity results } \\
\text { in symptoms (marked limitation) }\end{array}$ \\
IV & $\begin{array}{l}\text { Symptomatic at rest, increased discomfort with any } \\
\text { physical activity }\end{array}$
\end{tabular}

\subsubsection{Classifying severity of $\mathrm{HF}$}

Symptoms are used to classify the severity of effort intolerance in congestive HF. The New York Heart Association (NYHA) classification is recommended (Table 1). For common daily activities, use examples:

- Walking distance on level ground

- Class II - walk $>2$ blocks on the level

- Class III - walk $<1$ - 2 blocks

- Number of stairs climbed

- Class I - cope without symptoms

- Class II - climb 1 flight at normal pace

- Class III - climb $<1$ flight

- Tolerance of uphill

- Class I - cope without symptoms

- Class II - symptomatic on walking uphill

- Class III - symptomatic on any incline.

Limitations of the NYHA classification include:

- Ordinary physical activity is difficult to define and varies between individual patients and at different ages

- Mild symptoms do not imply minor cardiac dysfunction, and vice versa.

The NYHA functional class classification is useful to prognosticate, as functional capacity is a predictor of mortality.

It also provides a guide to the patient's previous best functioning so that therapy can be targeted at returning the patient to that level and better.

\subsubsection{Signs}

These include tachycardia, tachypnoea, raised jugular venous pressure, peripheral oedema, right-sided pleural effusion and hepatomegaly. Objective evidence of a structural and/or functional abnormality of the LV at rest (displaced LV apex beat, left-sided S3 gallop and cardiac murmurs).

\subsubsection{Documentation of HF-REF}

The echocardiogram provides immediate information on chamber size, ventricular function, LV wall thickness, valve structure and function, haemodynamics, pulmonary pressures and inferior vena caval congestion.

A patient presenting with HF requires an echocardiogram as part of the diagnostic assessment. While it is recognised that this depends on local availability, echocardiography should be arranged during the index hospital admission.

\section{General diagnostic tests in patients with suspected HF-REF \\ 4.1 Additional essential investigations}

These include electrocardiogram (ECG), chest X-ray and B-type natriuretic peptides (BNPs)/ $\mathrm{N}$-terminal pro-B-type natriuretic peptide (NT-ProBNP) assays.

\subsubsection{Resting ECG}

This may show sinus tachycardia ( $>100 \mathrm{bpm})$, bundle branch block patterns, heart block, previous myocardial infarction (MI), left ventricular hypertrophy, atrial fibrillation (AF), among other abnormalities. A normal ECG in all respects makes the diagnosis of HF-REF unlikely.

\subsubsection{Chest X-ray}

This should be considered to detect/exclude lung disease. It may also identify pulmonary congestion/oedema and is more useful in patients with suspected HF in the acute setting. The important findings in chronic HF-REF are increased cardiothoracic ratio $(>50 \%)$, right-sided pleural effusion, fluid in the fissures and upperlobe venous blood diversion.

\subsubsection{Natriuretic peptides}

Where the availability of echocardiography is limited, an alternative approach to the diagnosis of HF is to measure the blood concentration of BNPs. Two different assays are currently available: BNP and NT-ProBNP. A BNP $<100 \mathrm{pg} / \mathrm{ml}$ or NT-ProBNP $<300 \mathrm{pg} / \mathrm{ml}$ (in patients who present acutely) and BNP $<35 \mathrm{pg} / \mathrm{ml}$ or NT-ProBNP $<125 \mathrm{pg} / \mathrm{ml}$ in patients who present (with chronic symptoms) virtually exclude the diagnosis of HF-REF. These tests are also useful in the follow-up of treated patients to clarify future presentations or persistence of symptoms.

Routine biochemical and haematological investigations are also important to determine renal function and potassium levels, to exclude anaemia (which can mimic or aggravate HF) and to assess thyroid dysfunction (thyroid-stimulating hormone (TSH)), which may aggravate HF. They may also provide other useful information related to the aetiology of HF (e.g. elevated blood glucose levels, suggesting the diagnosis of diabetes).

\subsection{Further evaluation}

Patients with HF-REF who are referred to a cardiologist may be further evaluated with:

- ambulatory electrocardiographic monitoring (Holter)

- myocardial perfusion/ischaemia/viability imaging (echocardiography, cardiovascular magnetic resonance imaging (CMR), singlephoton emission computed tomography (SPECT) or positron emission tomography (PET))

- coronary angiography

- left and right heart haemodynamic assessments

- exercise testing

- genetic testing.

\section{Management of $\mathbf{H F}$ \\ 5.1 Objectives}

To make patients feel better; to reduce hospitalisations (new and recurrent); and to prolong survival. It is now recognised that preventing HF hospitalisation is important for patients and healthcare systems. 
5.2 Pharmacological therapies of HF-REF

\subsubsection{Symptomatic relief}

\subsubsection{Diuretics}

Diuretics are recommended to relieve dyspnoea and oedema in patients with signs and symptoms of congestion. Their effects on mortality and morbidity have, however, not been studied.

Loop diuretics produce a more intense and shorter diuresis than thiazides, which cause a more gentle and prolonged diuresis. Loop diuretics are usually preferred to thiazides in HF-REF.

The aim of using diuretics is to achieve and maintain euvolaemia (the patient's 'dry weight') with the lowest dose possible. This means that the dose must be adjusted, particularly after restoration of dry body weight, to avoid the risk of dehydration leading to hypotension and renal dysfunction. The diuretic dose may have to be reduced after angiotensin-converting enzyme inhibitors (ACEIs) are introduced. It is important to replace serum $\mathrm{K}^{+}$accordingly (1 $200 \mathrm{mg}$ potassium salt per $40 \mathrm{mg}$ furosemide). Potassium supplementation needs to be reduced in patients on concomitant MRAs. Intravenous (IV) diuretic should be used when there is marked volume overload and/ or pulmonary oedema.

Many patients can be trained to self-adjust their diuretic dose, based on monitoring of symptoms/signs of congestion and daily weight measurements. Patients should be encouraged to restrict fluid intake $(<1.51$ /day $)$.

\subsection{Diuretic resistance}

If a patient does develop resistance to a high dose of loop diuretic, the addition of a thiazide (hydrochlorothiazide) or thiazide-like (metolazone) diuretic (1 hour before administration of the loop diuretic) often results in a remarkable synergistic effect. These patients should ideally be referred, as large fluid shifts occur together with electrolyte changes. Careful patient and laboratory monitoring is necessary.

Refer to Table 2 for the recommended diuretics and their doses. Please note that metolazone is available in South Africa on Section 21.

\subsubsection{Digoxin}

Digoxin is recommended in patients on conventional therapy with symptomatic $\mathrm{HF}$ and rapid $\mathrm{AF}$ to slow the ventricular response rate.
Digoxin may be used in HF-REF and sinus rhythm if patients are still symptomatic on full tolerated background therapy to reduce the risk of HF hospitalisation.

Toxic drug levels can occur especially in the elderly, patients with poor renal function, in the presence of hypokalaemia, active coronary ischaemia and low body weight (recommended dose is $0.125 \mathrm{mg}$ at night). Always be aware when introducing other drugs of their possible interaction with digoxin metabolism. Digoxin levels should be monitored carefully and one should aim for a therapeutic blood level of $0.5-0.8 \mathrm{ng} / \mathrm{ml}$ taken 12 hours after the last dose.

\subsubsection{Symptomatic relief and mortality benefit}

Three neurohumoral antagonists, an ACEI (or angiotensin receptor blocker (ARB)), a beta-blocker (BB) and MRAs are fundamentally important in modifying the course of HF-REF and should be considered in every patient. The order of initiation between an ACEI and $\mathrm{BB}$ is not crucial and either can be started first.

\subsubsection{ACEIs}

An ACEI is recommended for all patients with HF-REF to reduce the risk of HF hospitalisation and premature death.

ACEIs occasionally cause worsening of renal function, hyperkalaemia, symptomatic hypotension, cough, and, rarely, angioedema. An ACEI should be used with caution in patients with inadequate renal function (serum creatinine $\geq 221 \mathrm{mmol} / \mathrm{l}$ or estimated glomerular filtration rate (eGFR) $\leq 30 \mathrm{ml} / \mathrm{min} / 1.73 \mathrm{~m}^{2}$ ). An ACEI should not be used in patients with an abnormal serum potassium level $>5.2 \mathrm{mmol} / \mathrm{l}$.

Therapy with ACEIs should always be initiated at a low dose (Table 3). If a patient develops symptomatic hypotension or pre-renal azotaemia, then decreasing or temporarily withdrawing the diuretic (24 - 48 hours) is preferable to discontinuing the ACEI.

Dosage titration:

- Following 24 - 48 hours of therapy, the patient should be contacted to ask about possible side-effects.

- Within one week of initiation of therapy, the patient should have the following checked: blood pressure (BP), urea, creatinine and electrolytes.

- If side-effects occur, treatment should be changed. Reduce diuretic dose if symptomatic hypotension or deteriorating renal function

Table 2. Doses of diuretics commonly used to treat HF (with and without a preserved ejection fraction, chronic and acute) ${ }^{[1]}$

\begin{tabular}{|c|c|c|c|c|}
\hline Diuretics & \multicolumn{2}{|c|}{ Initial dose (mg) } & \multicolumn{2}{|c|}{ Usual daily dose (mg) } \\
\hline \multicolumn{5}{|l|}{ Loop diuretics ${ }^{*}$} \\
\hline Furosemide & \multicolumn{2}{|l|}{$20-40$} & \multicolumn{2}{|l|}{$40-420$} \\
\hline Bumetanide & \multicolumn{2}{|l|}{$0.5-1.0$} & \multicolumn{2}{|l|}{$1-5$} \\
\hline Torasemide & \multicolumn{2}{|l|}{$5-10$} & \multicolumn{2}{|l|}{$10-20$} \\
\hline \multicolumn{5}{|l|}{ Thiazides $^{\dagger}$} \\
\hline Bendroflumethiazide & \multicolumn{2}{|l|}{2.5} & \multicolumn{2}{|l|}{$2.5-10$} \\
\hline Hydrochlorothiazide & \multicolumn{2}{|l|}{25} & \multicolumn{2}{|l|}{$12.5-100$} \\
\hline Metolazone & \multicolumn{2}{|l|}{2.5} & \multicolumn{2}{|l|}{$2.5-10$} \\
\hline Indapamide $^{\ddagger}$ & \multicolumn{2}{|l|}{2.5} & \multicolumn{2}{|l|}{$2.5-5$} \\
\hline Potassium-sparing diuretics ${ }^{s}$ & $+\mathrm{ACEI} / \mathrm{ARB}$ & -ACEI/ARB & $+\mathrm{ACEI} / \mathrm{ARB}$ & -ACEI/ARB \\
\hline Spironolactone/eplerenone & $12.5-25$ & 50 & 50 & $100-200$ \\
\hline Amiloride & 2.5 & 5 & $5-10$ & $10-20$ \\
\hline Triamterene & 25 & 50 & 100 & 200 \\
\hline
\end{tabular}




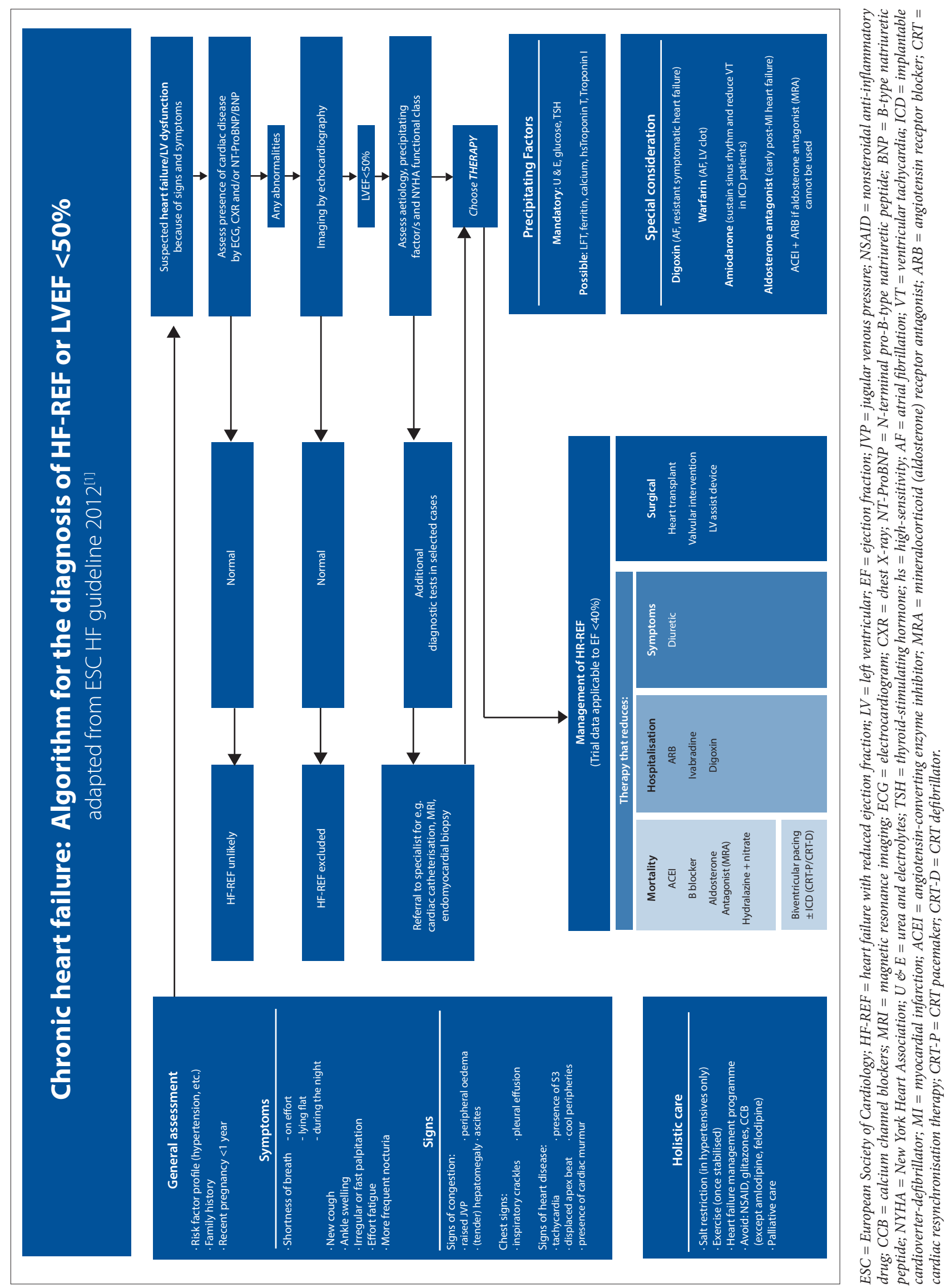


is found. Decrease potassium replacement if serum potassium is raised or decrease/stop potassium-sparing diuretics.

- An increase in serum creatinine need not require discontinuation of the ACEI.

- Dosage of ACEIs should be up-titrated over 2 - 4 weeks in ambulatory patients to target doses.

- ACEI cough (cough related to HF having been excluded), if severe, usually requires withdrawal of the ACEI.

\subsubsection{BBs}

$\mathrm{A} B \mathrm{BB}$ is recommended, in addition to an ACEI (or ARB if an ACEI is not tolerated), for all patients with HF-REF to reduce the risk of HF hospitalisation and the risk of premature death. The ideal candidate should not be on IV medications, should have no signs of marked fluid retention and be on stable doses of standard medication. Refer to Table 3 and note that only carvedilol and bisoprolol are widely available in South Africa.

\subsubsection{MRAs}

Spironolactone and eplerenone (Table 3) block receptors that bind aldosterone and other corticosteroids, and are best characterised as MRAs. Both can cause hyperkalaemia and worsening renal function, especially in the elderly.

An MRA is recommended for all patients with persisting symptoms (NYHA class II - IV) despite treatment with an ACEI (or an ARB if an ACEI is not tolerated) and a BB, to reduce the risk of HF hospitalisation and the risk of premature death.

Table 3. Evidence-based doses of disease-modifying drugs used in key randomised trials in HF (or after MI ${ }^{[1]}$

\begin{tabular}{|c|c|c|}
\hline & \multicolumn{2}{|c|}{ Dose (mg) } \\
\hline & Starting & Target \\
\hline \multicolumn{3}{|l|}{ ACEI } \\
\hline Captopril ${ }^{*}$ & 6.25 t.i.d. & 50 t.i.d. \\
\hline Enalapril & 2.5 b.i.d. & 10 - 20 b.i.d. \\
\hline Lisinopril $^{\dagger}$ & 2.5 - 5.0 o.d. & 20 - 35 o.d. \\
\hline Ramipril & 2.5 o.d. & 5 b.i.d. \\
\hline Trandolapril $^{*}$ & 0.5 o.d. & 4 o.d. \\
\hline \multicolumn{3}{|l|}{ Beta-blocker } \\
\hline Bisoprolol & 1.25 o.d. & 10 o.d. \\
\hline Carvedilol & 3.125 b.i.d. & 25 - 50 b.i.d. \\
\hline Metoprolol succinate (CR/XL) & $12.5 / 25$ o.d. & 200 o.d. \\
\hline Nebivolol $^{\ddagger}$ & 1.25 o.d. & 10 o.d. \\
\hline \multicolumn{3}{|l|}{$\mathrm{ARB}$} \\
\hline Candesartan & $4 / 8$ o.d. & 32 o.d. \\
\hline Valsartan & 40 b.i.d. & 160 b.i.d. \\
\hline Losartan ${ }^{\dagger, \neq}$ & 50 o.d. & 150 o.d. \\
\hline \multicolumn{3}{|l|}{ MRA } \\
\hline Eplerenone & 25 o.d. & 50 o.d. \\
\hline Spironolactone & 25 o.d. & 25 - 50 o.d. \\
\hline \multicolumn{3}{|c|}{ 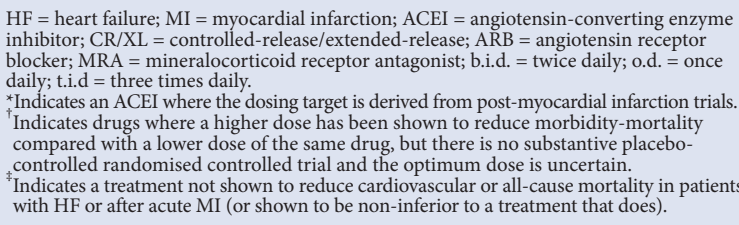 } \\
\hline
\end{tabular}

They should only be used in patients with adequate renal function (creatinine $\leq 221 \mathrm{mmol} / \mathrm{l}$ or $\leq 2.5 \mathrm{mg} / \mathrm{dl}$ or $\mathrm{eGFR} \geq 30 \mathrm{ml} / \mathrm{min} / 1.73 \mathrm{~m}^{2}$ ) and a normal serum potassium concentration $\left(\mathrm{K}^{+} 3.5-5.2 \mathrm{mmol} / \mathrm{l}\right)$; if either is used, serial monitoring of serum electrolytes and renal function is mandatory.

Potassium and creatinine should be monitored 5 days after starting treatment and be repeated 1 week later or on further titrations. The recommended dose for either spironolactone or eplerenone is: a starting dose of $25 \mathrm{mg}$ once daily and a target dose 25 - $50 \mathrm{mg}$ once daily.

Although spironolactone is first choice based on cost, patients who develop gynaecomastia should be switched to eplerenone. The combination of spironolactone and digoxin is synergistic for this complication.

\subsubsection{Symptom relief and uncertain mortality benefit}

\subsubsection{ARBs}

ARBs are recommended to reduce the risk of:

- HF hospitalisation and premature death in patients with HF-REF and who are unable to tolerate an ACEI because of cough (patients should also receive a BB and an MRA)

- HF hospitalisation in patients with HF-REF and who have persisting symptoms (NYHA class II - IV) despite treatment with an ACEI and a BB, and who are unable to tolerate an MRA.

\subsubsection{Ivabradine}

Ivabradine is a drug that inhibits the $\mathrm{I}_{\mathrm{f}}$ channel in the sinus node. Its only known pharmacological effect is to slow the heart rate in patients in sinus rhythm (it does not slow the ventricular rate in AF).

It should be considered to reduce the risk of HF hospitalisation in patients in sinus rhythm with an $\mathrm{EF} \leq 35 \%$ and a heart rate remaining at $\geq 70 \mathrm{bpm}$ and persisting symptoms (NYHA class II - IV) despite treatment with an evidence-based dose of $\mathrm{BB}$ (or maximum tolerated dose below that), an ACEI (or ARB) and an MRA (or ARB).

It may be considered to reduce the risk of HF hospitalisation in patients in sinus rhythm with an $\mathrm{EF} \leq 35 \%$ and a heart rate $\geq 70 \mathrm{bpm}$ who are unable to tolerate a BB. Patients should also receive an ACEI (or ARB) and an MRA (or ARB).

The South African Medicines Control Council (MCC) labelling, however, states that: 'Ivabradine is indicated in adults in sinus rhythm with mild to moderate (NYHA class II and III) symptomatic $\mathrm{HF}$ whose heart rate is $\geq 77 \mathrm{bpm}$ to reduce cardiovascular events (cardiovascular mortality or hospitalisation for worsening HF), in combination with standard therapy including $\mathrm{BB}$ or when $\mathrm{BB}$ are contraindicated or not tolerated'.

\subsubsection{Combination of hydralazine and nitrates}

This may be considered as an alternative to an ACEI or ARB, if neither is tolerated, to reduce the risk of HF hospitalisation and risk of premature death in patients with HF-REF. Patients should also receive a $\mathrm{BB}$ and an MRA.

It should be considered to reduce the risk of HF hospitalisation and risk of premature death in patients with HF-REF and persisting symptoms (NYHA class II - IV) despite treatment with a BB, ACEI (or ARB) and an MRA (or ARB).

Recommended dosages of the combinations:

- hydralazine 10 - $25 \mathrm{mg} 3$ times daily, increased to $75 \mathrm{mg} 3$ times daily

- isosorbide dinitrate $10 \mathrm{mg} 3$ times daily, increased to $40 \mathrm{mg} 3$ times a day 
- isosorbide mononitrate $10 \mathrm{mg}$ twice a day, increased to 20 - $40 \mathrm{mg}$ twice a day.

\subsection{Ventricular rate control in AF}

For ventricular rate control in patients with HF-REF and AF, a BB is preferred over digoxin, as the latter does not provide adequate rate control during exercise. The combination of digoxin and a $\mathrm{BB}$ is more effective than a BB alone in controlling the ventricular rate at rest.

Non-dihydropyridine calcium channel blockers (CCBs) verapamil and diltiazem are not recommended in patients with HF-REF as they increase mortality.

\subsection{Oral anticoagulants}

Warfarin is not routinely required in patients with HF-REF unless they have AF or LV mural thrombus.

\section{Non-pharmacological therapies of HF-REF}

6.1 Implantable cardioverter-defibrillator (ICD)

Approximately half of the deaths in patients with HF, especially in those with milder symptoms, occur suddenly and many, if not most, of these are related to ventricular arrhythmias.

Prevention of sudden death is therefore an important goal in HF. While the key disease-modifying neurohumoral antagonists mentioned earlier reduce the risk of sudden death, they do not abort it. Specific anti-arrhythmic drugs do not decrease this risk (and may even increase it). Therefore, ICDs play an important role in reducing the risk of death from ventricular arrhythmias and bradyarrhythmias.

\subsubsection{Primary prevention of sudden cardiac death} An ICD is recommended in a patient with symptomatic HF (NYHA class II - III) and an EF $\leq 35 \%$ despite $\geq 3$ months of treatment with optimal pharmacological therapy, who is expected to survive for $>1$ year with good functional status, to reduce the risk of sudden death. ICD implantation should be delayed for $>40$ days following MI, since LV function/EF may improve during this interval.

\subsubsection{Secondary prevention of sudden cardiac death}

ICDs reduce mortality in survivors of cardiac arrest and in patients with sustained symptomatic ventricular arrhythmias. Consequently, an ICD is recommended in such patients, irrespective of EF, with good functional status, a life expectancy of $>1$ year, and where the intent is to increase survival.

\subsection{CRT}

The indications for CRT are summarised in Tables 4 and 5 .

\subsection{Patients in permanent AF}

CRT pacemaker (CRT-P)/CRT defibrillator (CRT-D) may be considered in patients in NYHA functional class III or ambulatory class IV with a QRS duration $\geq 120 \mathrm{~ms}$ and an $\mathrm{EF} \leq 35 \%$, who are expected to survive with good functional status for $>1$ year, to reduce the risk of HF worsening, if the patient:

- requires pacing because of an intrinsically slow ventricular rate

- is pacemaker-dependent as a result of AV nodal ablation.

In patients with an indication for conventional pacing and no other indication for CRT: in patients who are expected to survive with good

Table 4. Recommendations for use of CRT where the evidence is strong - patients in sinus rhythm with NYHA functional class III and ambulatory class IV HF and a persistently reduced EF, despite optimal pharmacological therapy ${ }^{[1]}$

\begin{tabular}{l}
\hline Recommendations \\
\hline LBBB QRS morphology \\
CRT-P/CRT-D is recommended in patients in sinus rhythm with a QRS duration of $\geq 120$ ms, LBBB QRS morphology, \\
and an EF $\leq 35 \%$, who are expected to survive with good functional status for $>1$ year, to reduce the risk of HF \\
hospitalisation and risk of premature death \\
Non-LBBB QRS morphology \\
CRT-P/CRT-D should be considered in patients in sinus rhythm with a QRS duration of $\geq 150$ ms, irrespective of QRS \\
morphology, and an EF $\leq 35 \%$ who are expected to survive with good functional status for $>1$ year, to reduce the risk of \\
HF hospitalisation and the risk of premature death \\
CRT $=$ cardiac resynchronisation therapy; NYHA = New York Heart Association; HF = heart failure; EF = ejection fraction; LBBB = left bundle branch block; CRT-D = CRT defibrillator; \\
CRT-P = CRT pacemaker; RCT = randomised clinical trial. \\
${ }^{*}$ Level of evidence: A = data derived from multiple RCTs or meta-analyses; $B=$ data derived from a single RCT or large non-randomised studies; C $=$ consensus of opinion of the experts and/or \\
small studies, retrospective studies, registries.
\end{tabular}

Table 5. Recommendations for the use of CRT where the evidence is strong - patients in sinus rhythm with NYHA functional class II HF and a persistently reduced EF, despite optimal pharmacological therapy ${ }^{[1]}$

\begin{tabular}{l}
\hline Recommendations \\
\hline LBBB QRS morphology \\
CRT, preferably CRT-D, is recommended in patients in sinus rhythm with a QRS duration of $\geq 130$ ms, LBBB QRS \\
morphology, and an EF $\leq 30 \%$, who are expected to survive for $>1$ year with good functional status, to reduce the risk \\
of HF hospitalisation and the risk of premature death \\
Non-LBBB QRS morphology \\
CRT, preferably CRT-D, should be considered in patients in sinus rhythm with a QRS duration of $\geq 150$ ms, \\
irrespective of QRS morphology, and an EF $\leq 30 \%$, who are expected to survive for $>1$ year with good functional status, \\
to reduce the risk of HF hospitalisation and the risk of premature death \\
CRT = cardiac resynchronisation therapy; NYHA = New York Heart Association; HF = heart failure; EF = ejection fraction; LBBB = left bundle branch block; CRT-D = CRT defibrillator; \\
CRT-P = CRT pacemaker; RCT = randomised clinical trial.
\end{tabular}




\section{GUIDELINE}

functional status for $>1$ year, CRT should be considered in those in NYHA functional class II - IV with an $\mathrm{EF} \leq 35 \%$, irrespective of QRS duration, to reduce the risk of worsening of HF.

\section{Hypertension}

Hypertension is associated with an increased risk of developing HF; antihypertensive therapy markedly reduces the incidence of HF (with an exception of alpha-adrenoceptor blockers, which are less effective than other antihypertensives in preventing HF).

Negatively inotropic CCBs (i.e. diltiazem and verapamil) should not be used to treat hypertension in patients with HF-REF, and moxonidine should also be avoided in patients with HF-REF as it is associated with increased mortality.

If $\mathrm{BP}$ is not controlled with an ACEI (or ARB), a BB, MRA and a diuretic, then a choice from hydralazine/amlodipine/felodipine can be made. Short-acting nifedipine is contraindicated in HF-REF and no data are available on long-acting nifedipine.

\section{Heart transplantation}

Heart transplantation is an accepted treatment for end-stage HF Although controlled trials have never been conducted, there is consensus that transplantation - provided that proper selection criteria are applied - significantly increases survival, exercise capacity, quality of life, and return to work compared with conventional treatment.

Apart from the shortage of donor hearts, the main challenges in transplantation are the consequences of the limited effectiveness and complications of immunosuppressive therapy in the long term (i.e. antibody-mediated rejection, infection, hypertension, renal failure, malignancy and coronary artery vasculopathy).

There are four heart transplantation centres in South Africa: two in Cape Town (Christiaan Barnard Memorial and Groote Schuur hospitals), one in Johannesburg (Milpark Hospital) and one in Durban (Ethekwini Hospital and Heart Centre).

\subsection{Indications for heart transplantation}

\subsubsection{Absolute indications in appropriate patients}

These include:

- Haemodynamic compromise due to HF:

- refractory cardiogenic shock

- documented dependence on IV inotropic support to maintain adequate organ perfusion

- peak oxygen consumption per unit time $\left(\mathrm{VO}_{2}\right)<10 \mathrm{ml} / \mathrm{kg} / \mathrm{min}$ with achievement of anaerobic metabolism.

- Severe symptoms of ischaemia that consistently limit routine activity and are not amenable to coronary artery bypass surgery or percutaneous coronary intervention.

- Recurrent symptomatic ventricular arrhythmias refractory to all therapeutic modalities.

\subsubsection{Relative indications}

- Peak $\mathrm{VO}_{2} 11$ - $14 \mathrm{ml} / \mathrm{kg} / \mathrm{min}$ (or 55\% predicted) and major limitation of the patient's daily activities

- Recurrent unstable ischaemia not amenable to other interventions

- Recurrent instability of fluid balance/renal function not due to patient non-compliance with medical regimen.

\subsubsection{Insufficient indications}

- Low LVEF

- History of functional class III or IV symptoms of HF

- Peak $\mathrm{VO}_{2}>15 \mathrm{ml} / \mathrm{kg} / \mathrm{min}$ (and $>55 \%$ predicted) without other indications.

\subsubsection{Haemodynamic criteria for evaluation of candidates for} transplantation

- Pulmonary artery hypertension and elevated pulmonary vascular resistance (PVR) should be considered as a relative contraindication to cardiac transplantation when the PVR is $>5$ Wood units or the pulmonary vascular resistance index (PVRI) is $>6$ or the transpulmonary gradient (TPG) exceeds $16-20 \mathrm{mmHg}$

- If the PAS exceeds $60 \mathrm{mmHg}$ in conjunction with any 1 of the preceding 3 variables, the risk of right $\mathrm{HF}$ and early death is increased

- If the PVR can be reduced to $<2.5$ with a vasodilator but the systolic BP falls $<85 \mathrm{mmHg}$, the patient remains at high risk of right $\mathrm{HF}$ and mortality after heart transplantation.

\section{Exercise training}

Several systematic reviews and meta-analyses of small studies have shown that physical conditioning by exercise training improves exercise tolerance, health-related quality of life, and HF hospitalisation rates in patients with $\mathrm{HF}$.

\section{Organisation of care and multidisciplinary management programmes}

The goal of management of HF is to provide a 'seamless' system of care, embracing both the community and hospital, to ensure that the management of every patient is optimal, from the beginning to the end of their healthcare journey.

To achieve this goal, other services, such as cardiac rehabilitation and palliative care, must be integrated into the overall provision for patients with HF.

Fundamental to the delivery of this complete package of care are multidisciplinary management programmes designed to improve outcomes through structured follow-up with patient education, optimisation of medical treatment, psychosocial support, and improved access to care.

\section{References}

1. McMurray JJV, Adamopoulos S, Anker SD, et al.; on behalf of the European Society of Cardiology. ESC guidelines for the diagnosis and treatment of acute and chronic heart failure 2012. Eur Heart J 2012;33(14):1787-1847. [http://dx.doi.org/10.1093/eurhearti/ehs104]

2. Bristow MR, Saxon LA, Boehmer J, et al. Cardiac-resynchronization therapy with or without an implantable defibrillator in advanced chronic heart failure. N Engl J Med 2004;350(21):21402150. [http://dx.doi.org/10.1056/NEJMoa032423]

3. Cleland JG, Daubert JC, Erdmann E, et al. The effect of cardiac resynchronization on morbidity and mortality in heart failure. N Engl J Med 2005;352(15):1539-1549. [http://dx.doi.org/10.1056/ NEJMoa050496]

4. Moss AJ, Hall WJ, Cannom DS, Klein H, et al. Cardiac-resynchronization therapy for the prevention of heart-failure events. N Engl J Med 2009;361(14):1329-1338. [http://dx.doi. rg/10.1056/NEJMoa0906431]

5. Tang AS, Wells GA, Talajic M, et al. Cardiac-resynchronization therapy for mild-tomoderate heart failure. N Engl J Med 2010;363(25):2385-2395. [http://dx.doi.org/10.1056/ NEJMoa 1009540] 